\title{
Article \\ Using Homemade Pressure Device to Improve Plantar Pressure-A Case Study on the Patient with Lower Limb Lymphedema
}

\author{
Jong-Chen Chen ${ }^{1, *}$, Yao-Te Wang ${ }^{1}(\mathbb{D})$ and Ying-Sheng Lin ${ }^{2}$ \\ 1 Information Management, National Yunlin University of Science and Technology, Yunlin 64002, Taiwan; \\ d10123001@gemail.yuntech.edu.tw \\ 2 National Taiwan University Hospital Yunlin Branch, Yunlin 640203, Taiwan; Y01924@ms1.ylh.gov.tw \\ * Correspondence: jcchen@yuntech.edu.tw; Tel.: +886-921-717-966
}

Citation: Chen, J.-C.; Wang, Y.-T.; Lin, Y.-S. Using Homemade Pressure Device to Improve Plantar

Pressure-A Case Study on the Patient with Lower Limb Lymphedema. Appl. Sci. 2021, 11, 9629. https://doi.org/10.3390/ app11209629

Academic Editor: Robert Koprowski

Received: 14 September 2021

Accepted: 11 October 2021

Published: 15 October 2021

Publisher's Note: MDPI stays neutral with regard to jurisdictional claims in published maps and institutional affiliations.

Copyright: () 2021 by the authors. Licensee MDPI, Basel, Switzerland. This article is an open access article distributed under the terms and conditions of the Creative Commons Attribution (CC BY) license (https:// creativecommons.org/licenses/by/ $4.0 /)$.

\begin{abstract}
Feet play a very important and indispensable role in people's lives. Patients with lymphedema often suffer from collapsed (or even deformed) foot arches as a result of lower extremity edema. This result will change the normal pressure distribution on the soles of their feet, which will affect their mobility and physical health. When the patient does not know that the distribution of pressure on the sole of the foot has changed significantly, the deformation of the sole of the foot will become severe. In response to this problem, this research team hopes to use a set of self-made sensor insoles to help to understand the plantar pressure points in different situations or actions. The subject invited in this study was a patient with lower extremity edema. The entire study was carried out with the consent of the patient, the guidance of the physician and the approval of the Ethics Committee of National Taiwan University Hospital (No: 201805068 RINB, date: 18 June 2018). This study uses this self-made sensor insole to analyze the plantar pressure distribution of the patient before and after the operation of lower extremity edema. The results show that the operation can effectively improve the high foot pressure in the center and rear of the foot area during different sports (standing, walking and biking). This not only increases its stability when standing and walking, but also significantly and effectively improves its walking speed and step distance.
\end{abstract}

Keywords: arch insole; lymphedema patient; plantar pressure; $t$-test; textured insole

\section{Introduction}

Feet play a very important and indispensable role in people's lives. This is because on the one hand they allow us to move freely, and on the other hand they bear the ground reaction force caused by the weight. The arches of the foot play a decisive role in buffering the impact and pressure on the body when people stand or walk, etc. [1,2]. If a person's arch collapses, he (she) may walk awkwardly or even experience pain. Abnormal pressure may lead to foot injuries. However, whether it is excessive plantar pressure or insufficient plantar pressure bearing, it may lead to the formation of plantar ulcers [3]. It is generally believed that one can more or less directly or indirectly understand the medical cause of the patient's disease through the signal condition of the foot pressure [4]. There is another school of scholars who proposed that plantar pressure can be used to assess and treat the related pain problems of foot patients $[5,6]$. Some scholars have further argued that there is a clear positive correlation between neuropathic foot ulcers and increased plantar pressure $[7,8]$. However, some scholars argue that the increase in BMI is closely related to the peak pressure of the midfoot, and that a too-high peak midfoot can further cause plantar injury [9]. Summarizing the above literature, we can roughly say that if the plantar pressure is evenly distributed, it can effectively reduce foot injury [10].

Lymphedema refers to the interaction of soft tissue fluid and infection caused by the obstruction of lymphatic drainage in certain parts of the body, leading to the proliferation of 
subcutaneous fibrous connective tissue or fat sclerosis. When the fascia and subcutaneous soft tissue are infected, it can cause the skin to rupture and further cause the death of part of the body's soft tissues. The spread of this disease is very rapid. It is considered to be a sudden onset disease. More specifically, necrotizing fasciitis, which is considered a disease, causes rapid local tissue necrosis and life-threatening severe sepsis.

Long-term lymphedema (especially if not handled properly) can lead to various complications, such as infection, disfigurement, pain and disability, and even fatal injuries. Furthermore, a patient with lymphedema is more likely to have foot arch deformity due to lower extremity edema or lack of protection of the arch. This result might cause the patient to have plantar discomfort, or even other parts of the body will be further damaged or collapsed. What is more serious is that these patients do not like sports because of uncomfortable soles. The cross-effects of the above-mentioned different causal relationships result in severe overweight in patients. In short, due to the lack of arch protection, lower limb edema is more harmful to the body than edema in other parts of the body. In addition, lymphedema is more likely to affect the dynamic performance of the whole body when walking due to edema, and the patient may even have a smaller step distance or a slower pace [11]. We all know that lymphedema is not a disease that can be summarized and treated simply. The entire treatment of lymphedema, from its cause to possible treatment and even post-treatment management, is a long-term process. In this lengthy process, it is very important to collect and understand the patient's sole condition in a timely and appropriate manner. If a general hospital physical examination of lower extremity edema is adopted, although it can reflect the actual condition of the patient, it is a relatively expensive and time-consuming method. If an economical and non-invasive method of detecting the plantar pressure of patients can be proposed, on the one hand, the patients can be informed of their plantar pressure in an early and timely manner, and on the other hand, the doctor can understand the patient's condition in order to provide appropriate diagnosis and treatment. With the efforts of both parties, the patient's condition may be improved to some extent by receiving more timely information. Some scholars suggest using a customized high-resolution pressure sensor to perform a comprehensive pressure analysis to establish a compression therapy based on efficacy [12].

It is stated here that the above-mentioned relatively simple detection method only aims to provide patient information in a relatively simple and convenient way. As far as accurate and correct medical treatment is concerned, the entire course of treatment still needs to rely on the hospital's complete testing information. This study hopes to build a set of self-made pressure sensor insoles to provide patients with lower extremity edema the means to understand their plantar pressure conditions at different time periods and exercise conditions in a timely manner. This study involved a patient with lower extremity edema. With the consent of the patient, physician and ethics committee, this study used this self-made induction insole to examine the patient's lower extremity edema before and after operation with respect to the patient's plantar pressure distribution during different exercises.

\section{Subject, Device, Data Collection and Experiments}

As mentioned earlier, this study hopes to detect the plantar pressure of overweight patients with lower extremity edema through a set of self-made pressure sensor insoles. This set of self-made pressure sensor insoles is divided into left and right feet, and each insole is equipped with six pressure resistance sensors. In this study, a patient with severe edema of the lower extremities was invited to perform plantar pressure tests before and after surgery for the edema of the lower extremities under the guidance of the attending physician. This part was mainly to understand the subtle changes in the plantar pressure of the patient before and after the operation. In order to understand the foot pressure of the patient in different daily activities, this study further invited the subject to stand, walk and ride a bicycle. Finally, in the hope of improving the patient's foot pressure, this study tried to use insoles of different heights and arch insoles to explore their impact on the patient's foot pressure. 
In the following, we first introduce the characteristics of the invited participant. Then, we explain the induction insoles, insoles of different heights and arch shoes specially made for this patient. After that, we explain the procedures for data collection. Finally, we briefly discuss the experimental methods.

\subsection{Subject}

The participant in this study was a patient with lower extremity edema due to necrotizing fasciitis. The following is his personal information: 56 years old, height $166 \mathrm{~cm}$, weight $130 \mathrm{~kg}$, BMI 47.2, does not smoke or drink, fat metabolism is obvious, lymphedema recurrence, diabetes, high blood pressure, kidney deficiency, poor vision, etc. As mentioned earlier, the entire study was carried out with the knowledge of the patient, the guidance of the physician and the consent of the ethics committee. In addition to the aforesaid symptoms of lymphedema, the subject had also suffered from severe flat feet problems.

\subsection{Device}

As mentioned earlier, the subject of this study is a patient with severe lower extremity edema with flat feet (Figure 1a). In order to understand his plantar pressure, this study designed a pair of self-made pressure sensor insoles specifically for his plantar pressure distribution. The pair of self-made pressure sensor insoles has left and right feet, and each insole is equipped with six pressure resistance sensors.

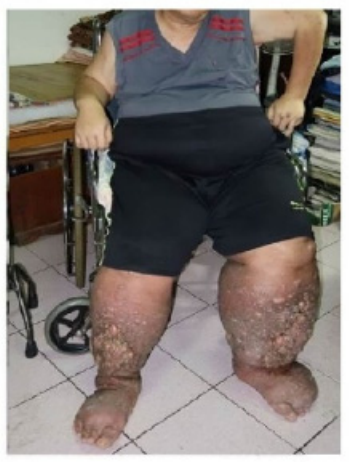

(a)

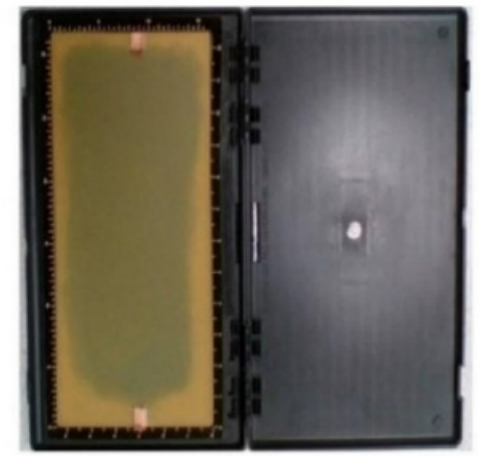

(b)

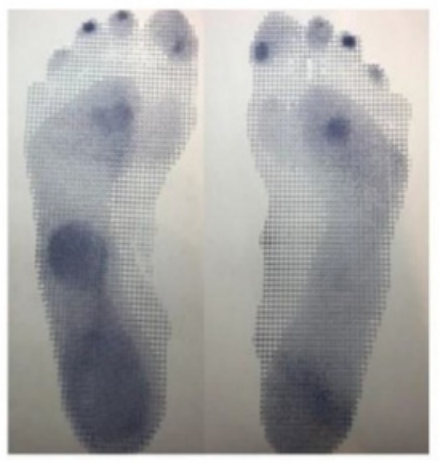

(c)

Figure 1. (a) The patient. (b) An MP-5 ink footprint device. (c) A patient's footprint image obtained from the MP-5 ink footprint device.

In order to effectively grasp the patient's foot information, the position of the six sensors on each foot is very important. In this study, the MP-5 ink footprint device (Figure 1b) was used to capture patient footprints (Figure 1c). In this footprint, the deeper the mark is, the higher the pressure in that area. Ink footprint is a cheap, simple and non-invasive method. The footprints obtained in this way can not only provide for the study of foot structure, but can also be used to diagnose pathological conditions of flat feet $[13,14]$.

According to the footprint, it was observed that the patient's greatest pressure was on the hind feet, and the second largest pressure was on the hallux and forefoot. After discussing with the doctor, the research team decided to place the six sensors on each foot as shown in Figure 2a: hallux (L1, R1), forefoot (L2, L3, R2, R3), midfoot (L4, L5, R4, R5) and hind feet (L6, R6). Figure $2 b$ shows a pair of pressure-sensing shoes designed by our team for the subject. 


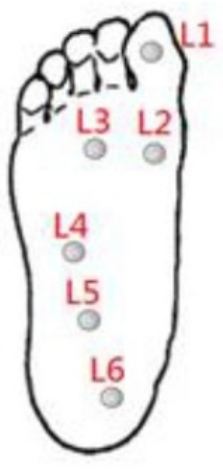

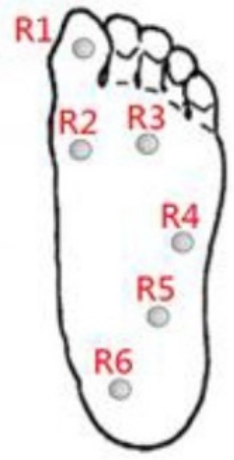

(a)

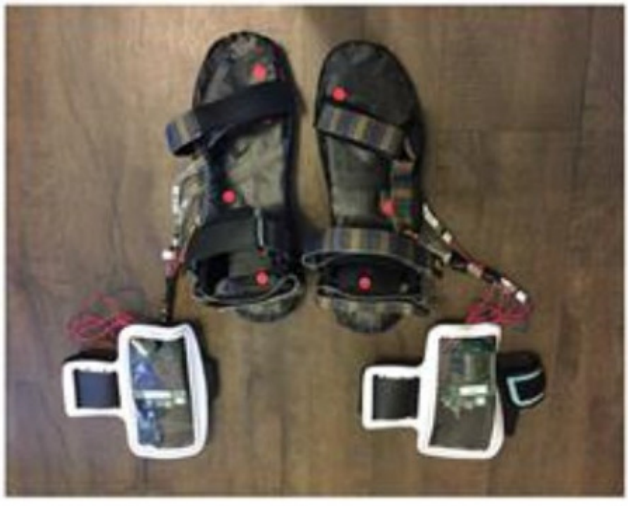

(b)

Figure 2. (a) The positions of the sole where the sensors are placed. (b) A pair of homemade pressure insoles and shoes.

In addition, the subject had a significant collapse of the soles of his feet due to edema of his lower extremities (the so-called flat family phenomenon). The research team hoped to use different height foot pads and arches to explore whether they can effectively improve plantar distribution. In response to this problem, the research team used a 3D printer to design three insoles of different heights $(0 \mathrm{~mm}, 3 \mathrm{~mm}$ and $6 \mathrm{~mm}$ ); each insole consists of 18 identical texture granules on the sole [15], as shown in Figure 3. In addition, an artificial arch support $(7 \mathrm{~cm}$ long, $1.5 \mathrm{~cm}$ wide and $1.5 \mathrm{~cm}$ high) was made for each foot.

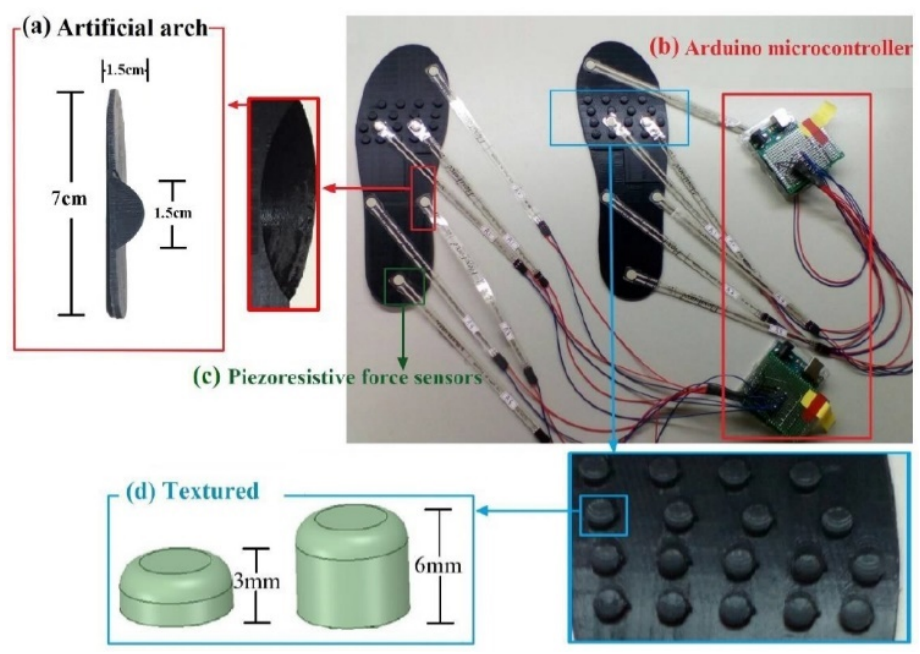

Figure 3. (a) An artificial arch support. (b) Arduino microcontroller. (c) Piezoresistive force sensors. (d) Textured granule.

\subsection{Experiments}

The experiments in this study are divided into two types. First of all, during the study period of this study, under the diagnosis of clinicians, the patient with lymphedema needed to undergo left foot fibrous tissue resection, right foot fibrous tissue resection and intra-abdominal lymph node transfer at different times. The research team took this opportunity to explore the changes in plantar pressure before and after the patient underwent surgery. During the experiment, the subject was invited to wear homemade insoles with no texture height and no arch. Under the guidance and supervision of the doctor, the subject performed three experiments of standing, walking and riding a bicycle before and after the operation (Figure 4). 


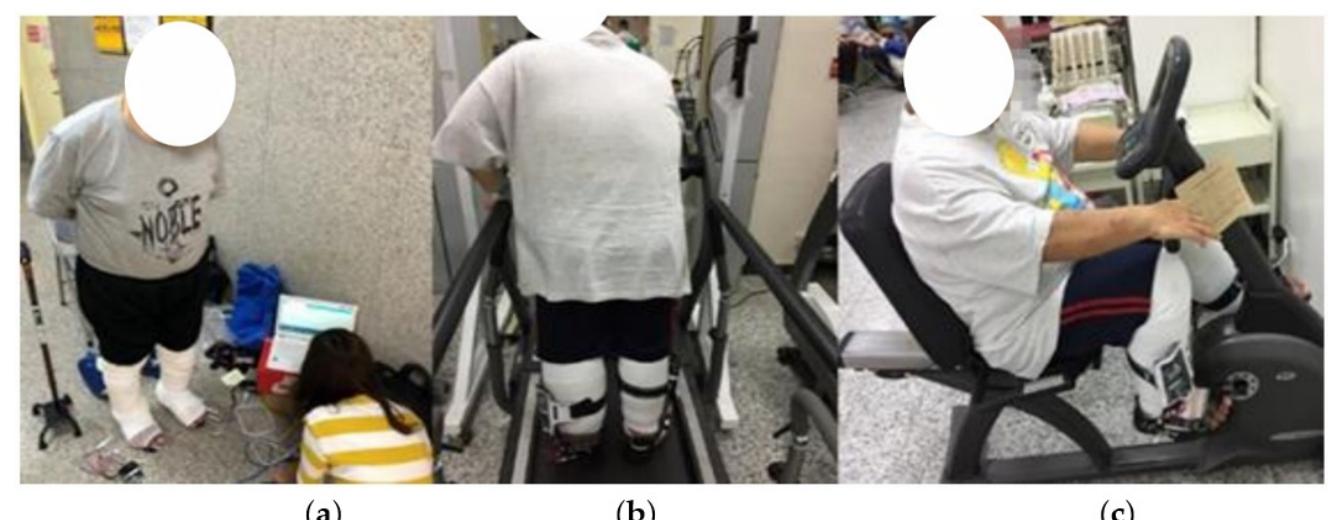

(a)

(b) (c)

Figure 4. Experiments of standing, walking and riding a bicycle. (a) Standing. (b) Walking. (c) Riding a bicycle.

The second type of experiment included investigating the effects of using different textured height insoles and foot arches on the plantar pressure when the patient underwent surgery. This experiment was performed five months after the surgery. During the course of the experiment, similarly, under the guidance and supervision of the physician, the patient with lymphedema was invited to wear the three different heights of the foot pressure insole (Figure 3) designed with the arch to explore the impact on the patient's plantar pressure. Because the forefoot was the largest area of foot during the stance phase, we only placed textured granules under the forefoot (18 textured granules of the same height and size). The artificial arch support was placed on the medial midfoot. The patient was requested to step directly on the specially designed pressure insole without shoes on.

\subsection{Data Collection Procedure}

All the data collected in this study were analyzed by $t$-test (SPSS statistical software). When the $p$ value is less than 0.05 , it indicates that there is a significant difference between the experimental group data and the control group data. In addition to the $p$-value, all experiments in this study simultaneously used three values: average pressure, peak pressure (PP) and standard deviation (STD) for analysis. MP represents the average plantar pressure that fluctuates up and down during the data collection process. PP is the maximum instantaneous plantar pressure value. STD represents the fluctuation of the pressure obtained at each pressure point relative to the average pressure during the data collection period.

\section{Results}

\subsection{Effect of Operation of Lower Extremity Edema on the Plantar Pressure Distribution}

This experiment attempts to shed light on the changes in the plantar pressure of the subject in standing, walking and biking after undergoing lymphedema surgery. In this experiment, the subject was asked to wear foot pressure-sensing shoes specially designed for him by the research team, and perform standing, walking and biking movements. Considering the physical condition of the test subject, the test time for each action was about 1 to $3 \mathrm{~min}$, depending on the types of experiments. After each experiment, the subject had a rest for about $10 \mathrm{~min}$.

\subsubsection{Standing}

The results show that, in terms of MP value (Figure 5), 6 out of 12 detection points on the left and right feet show significant differences, namely the left foot point L4 (from 395 to 121) and the left foot point L5 (from 278 to 138), right foot point R1 (from 86 to 12), right foot point R4 (from 554 to 147), right foot point R5 (from 1139 to 135) and right foot point R6 (from 1069 to 140). When the data was further analyzed, it could be shown that both the left foot and the right foot had relatively large improvements in the midfoot and hindfoot areas. 

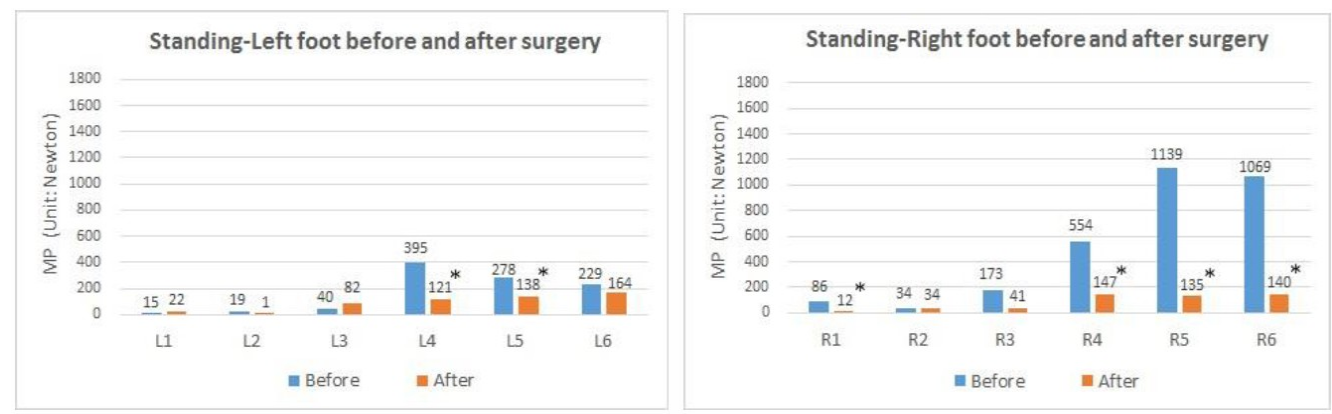

Figure 5. Standing MP in the lymphedema patient's foot before and after surgery. ${ }^{*} p$-value $<0.05$ indicates that a significant difference exists.

What is more special is that the subject's right foot improved more significantly than the left foot. This is because the subject's body is tilted to the right due to the serious edema of the left foot. This can be seen from the fact that the thumb pressure on the right side of the patient is greater than that on the left side. As a result, the sole of the right foot was relatively high before the operation. Because of this, the degree of improvement in plantar pressure after surgery is relatively large. What is more commendable is that if you further compare the MP value of the subject after the operation, we can see that the pressure value of the corresponding point on the left and right feet of the subject after the operation is roughly the same. This result shows that the whole operation is quite successful in terms of the subject's standing movement.

The following analysis is based on the maximum foot pressure PP. The maximum value represents the maximum value it may produce during the test. This value may be of little significance to people with normal soles. However, for a person with lower extremity edema, this value may cause irreversible damage. The results show that in terms of PP value, the left foot point L4 and point L6 and the right foot point R3 to R6 all show significant improvement (Figure 6). This value again confirms two things. The PP of the midfoot and hindfoot of the first subject is significantly improved due to surgery; the PP on the right of the second subject has a greater degree of improvement than the left.
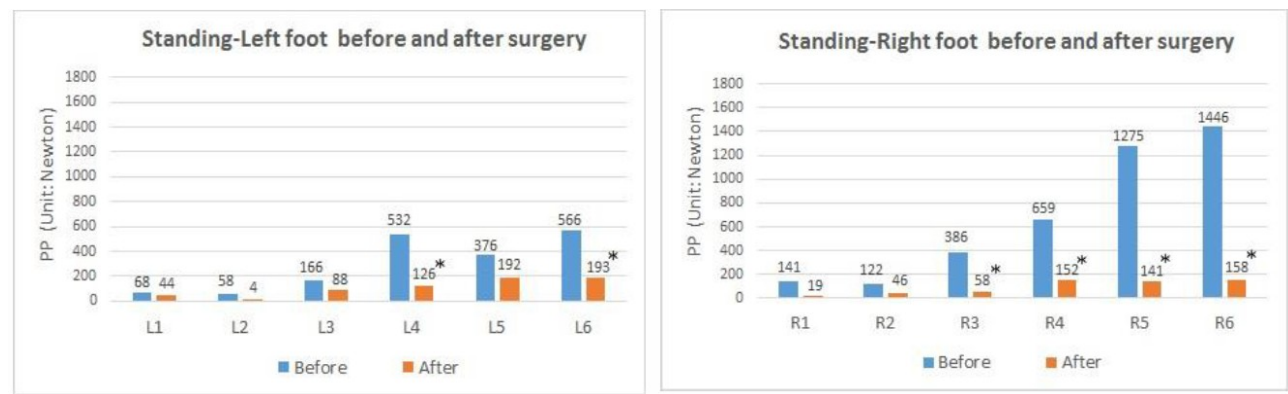

Figure 6. Standing PP in the lymphedema patient's foot before and after surgery. ${ }^{*} p$-value $<0.05$ indicates that a significant difference exists.

Finally, this study used the STD value (Figure 7) to analyze the patient's plantar pressure changes. This value indicates the change in the pressure of the patient within about 1-2 min of standing. In this study we interpret this change as the stability of the patient. The results showed that the STD value of L1 ranges from \pm 12.88 to \pm 1.51 , L2 ranges from \pm 14.03 to \pm 0.91 , L3 ranges from \pm 41.59 to \pm 7.75 , L4 ranges from \pm 75.44 to \pm 5.54 , L5 ranges from \pm 50.89 to \pm 3.49 and L6 ranges from +94.47 to \pm 1.92 . Obviously, the STD value of each point on the left foot has been significantly improved. Similarly, the STD value of each point on the right foot is also significantly reduced. Based on the above results, regardless of the MP, PP and STD values, postoperative foot pressure and 
body stability during standing were significantly improved, especially in the midfoot and hindfoot areas.
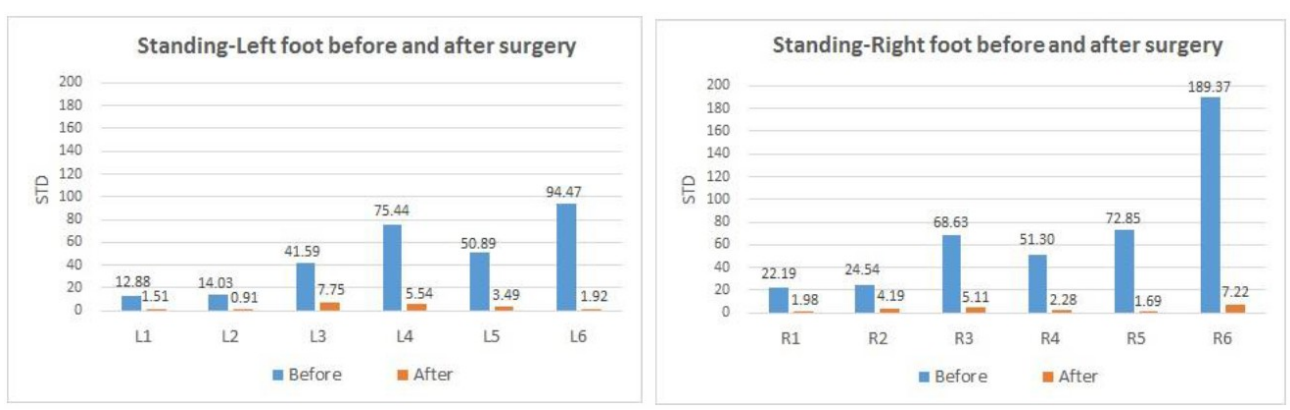

Figure 7. Standing STD in the lymphedema patient's foot before and after surgery.

\subsubsection{Walking}

Contrary to the previous section of the static standing test, this experiment mainly involved a dynamic walking test. As mentioned earlier, at a rate of 12 data collections per second, the research team invited the patient to walk for nearly three minutes at a speed of $1 \mathrm{~km}$ per hour on a walking machine. This experiment first removes the unstable noise data at the beginning of the experiment, and then officially starts collecting data. Figure 8 shows the pressure changes in the left and right feet before and after surgery while the patient was walking. A significant improvement can be seen from the values of the various pressure points before and after the patient's surgery. The curve of the pressure value at each point consisted of a steep mountain valley before surgery and a relatively gentle mountain valley after surgery. By referring to the maximum foot pressure PP value obtained during walking, except for the two points of the left foot L1 (hallux) and L6 (rearfoot), every other point of the left and right feet showed a significant improvement after surgery (Figure 9).

(a) Left foot
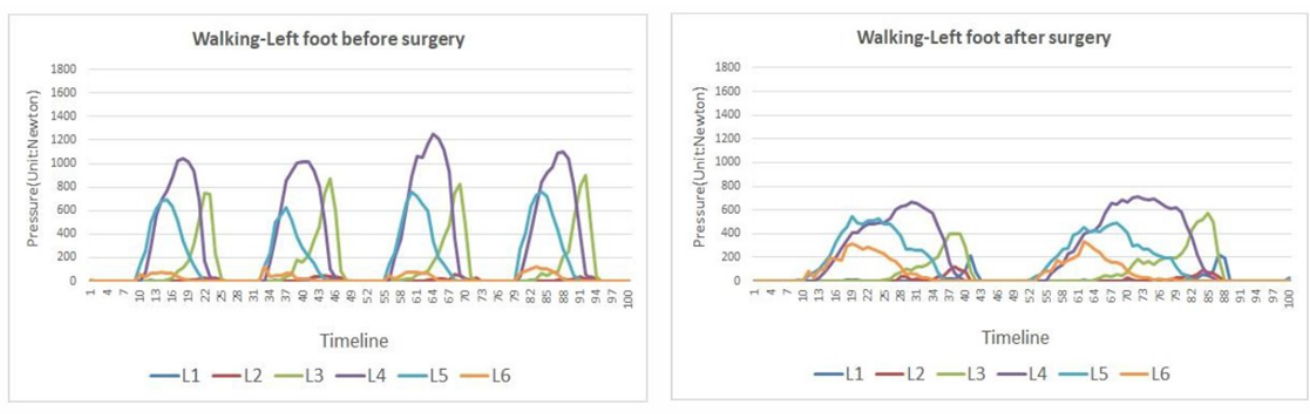

(b) Right foot
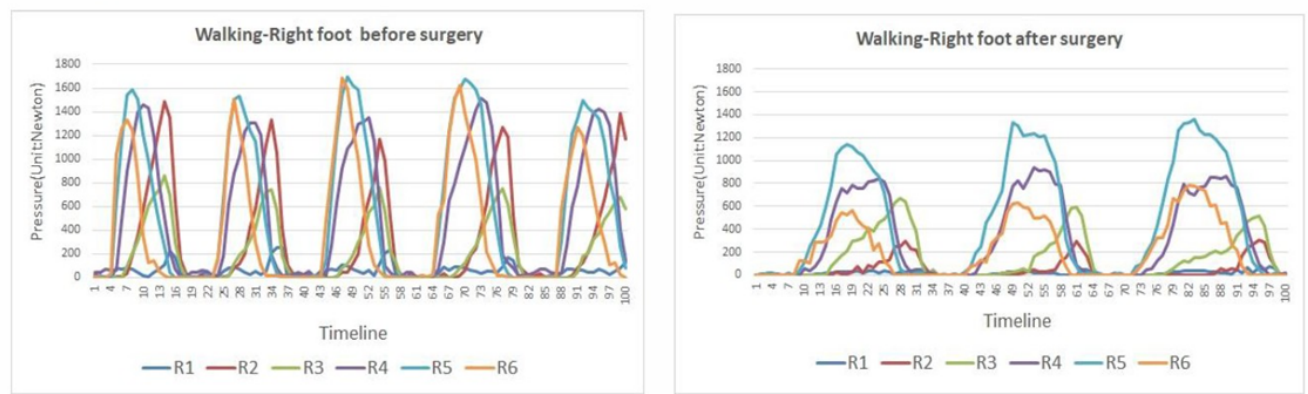

Figure 8. Walking pressure of the foot of the lymphedema patient before and after surgery. (a) Left foot. (b) Right foot. 

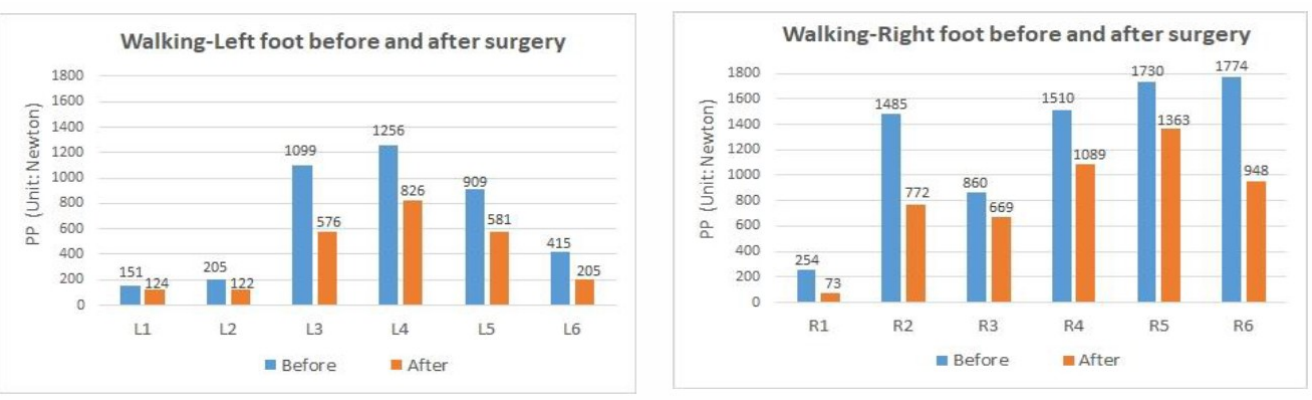

Figure 9. Walking PP in the lymphedema patient before and after surgery.

An interesting result is that not only has the patient's moving step distance after surgery doubled from before the surgery, but the walking speed has also increased by half. In other words, the patient moves at a slow speed with a short step before the operation, and moves at a relatively fast and large step after the operation. On the whole, in terms of walking, the patient regained a better pace after the operation due to changes in foot pressure. Based on the above experimental results, the operation not only effectively improved the high plantar pressure of the patient with lymphedema, but also effectively improved his pace and distance.

\subsubsection{Riding a Bicycle}

In the following experiment, the subject was invited to ride a rehabilitation bicycle for three minutes. Similar to the walking experiment, this is another dynamic test experiment. Figure 10 clearly shows the relationship between the actual contact surface of the sole and the midfoot mass (L4, L5, R4 and R5). Regardless of whether it is the left foot or the right foot, among the six detection points of each foot of the subject, the pressure in the four areas of the midfoot is still relatively high.

(a) Left foot
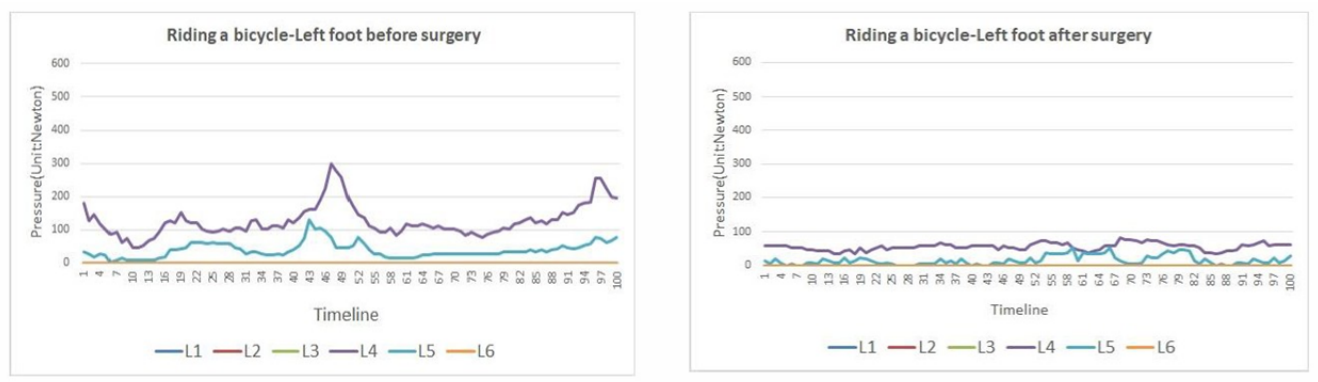

(b) Right foot
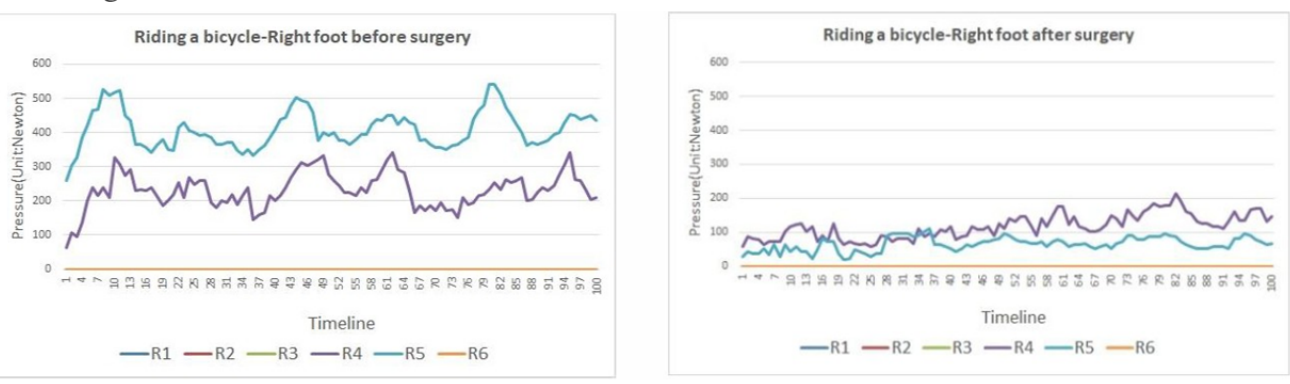

Figure 10. Pressure from riding a bicycle in the feet of the lymphedema patient before and after surgery. (a) Left foot. (b) Right foot.

Similar to the previous results, the pressure on the right foot of the patient before the operation was greater than that of the left foot, and its fluctuation range was also relatively large. After the operation, when the patient pedaled a bicycle with both feet, the pressure 
on the midfoot was significantly improved. However, the pressure on the right foot is still greater than that on the left foot, and there are some slight fluctuations. This may be because the edema of the left foot of the patient is more serious than the edema of the right foot before the operation, and the patient tries to use the right foot to replace the function of the left foot.

In terms of PP, as clearly seen in Figure 11, the value of the L4 point of the left foot decreased from 298 to 107, and the value of the L5 point decreased from 131 to 25. Similarly, the value of the R4 point of the right foot decreased from 400 to 215, while, in contrast, the value of the R5 point decreased from 598 to 136 . These results indicated that surgery can effectively improve the problem of high foot pressure in the midfoot block caused by cycling.
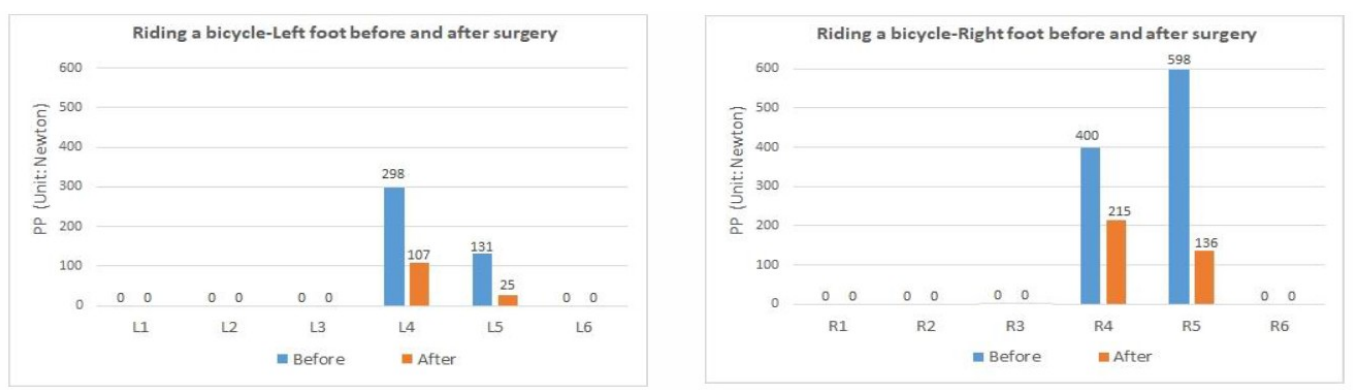

Figure 11. PP from riding a bicycle in the lymphedema patient before and after surgery.

\subsection{Effects of Using Textured Insoles of Different Heights and Artificial Arch on Plantar Pressure Distribution}

Five months after the subject had undergone all operations (when the patient had reached a fully recovered state), the research team further invited the patient to stand on an artificial foot arch and three different height textured insoles to explore whether the use of these assistive devices can improve plantar pressure distribution. For the design and description of these assistive devices, please refer to Section 2.3.

\subsubsection{Using Artificial Arches}

In this experiment, an artificial arch was placed in the midfoot area of the insole, and the patient was invited to stand on the footpad for one minute. The results demonstrated that the artificial arch added to the foot allowed the patient's left foot to have a significant improvement in MP values (Figure 12), especially for the areas of the midfoot and rearfoot (L4, L5 and L6). For the right foot, there was a significant improvement in the midfoot and rearfoot areas (R4, R5 and R6). The above results again demonstrated that the artificial arch was effective in helping to alleviate the collapse problem, and it even provided the patient with a considerable degree of assistance.
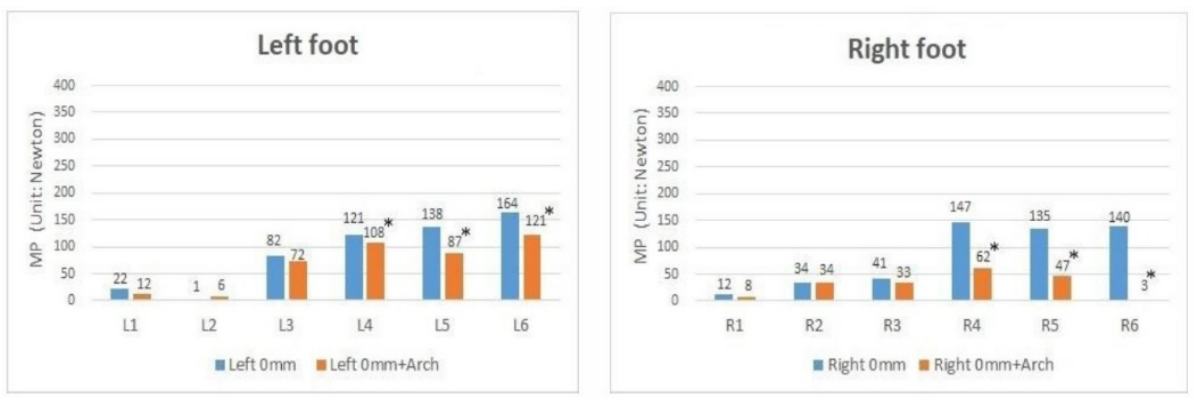

Figure 12. MP — effect of using an artificial arch in the lymphedema patient. * $p$-value $<0.05$ indicates that a significant difference exists.

From the perspective of PP (Figure 13), the foot pressure of the midfoot and hindfoot of each foot is significantly improved. This result is very similar to the result from MP. It is 
worth mentioning that the thumb toe of the left foot has improved significantly. This result is quite important from a certain point of view. We know that the degree of edema in the left foot of the subject is relatively serious. In other words, his left foot is relatively fragile and easily injured. If the weaker PP value can be improved, the possibility of instantaneous injury can be reduced.
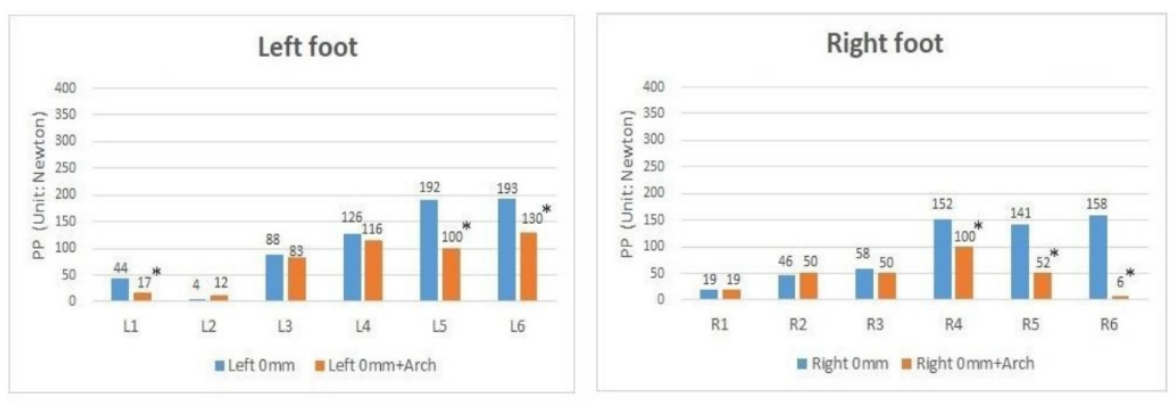

Figure 13. PP—effect of using an artificial arch in the lymphedema patient. ${ }^{*} p$-value $<0.05$ indicates that a significant difference exists.

Finally, this study uses the results of STD to explore patient stability. The results showed that the patient's STD value (Figure 14) decreased from \pm 7.75 to \pm 2.14 in L3, from \pm 5.54 to \pm 1.64 in L4, from \pm 5.11 to \pm 2.9 in $R 3$, from \pm 1.69 to \pm 0.87 in $R 5$ and from \pm 7.22 to \pm 1.07 in R6. With the aforesaid results in mind, the use of artificial arches not only reduced high foot pressure but also effectively improved the body stability of the subject.
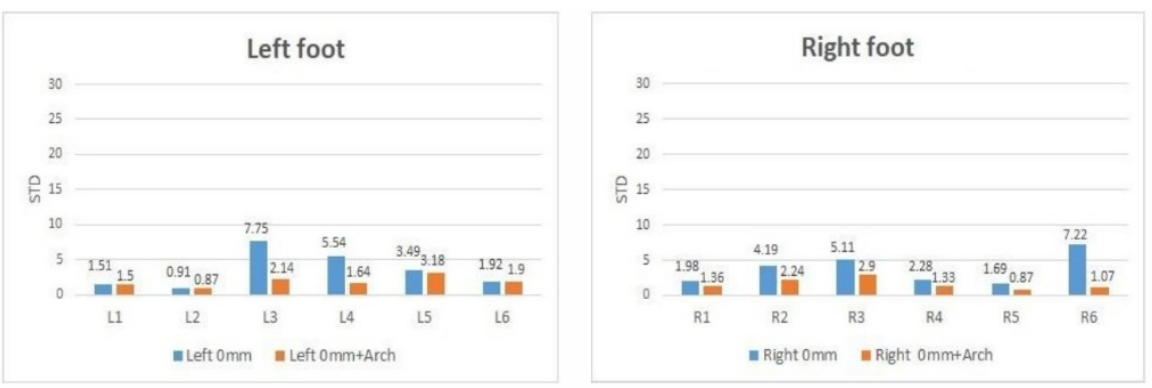

Figure 14. STD—effect of using an artificial arch in the lymphedema patient.

\subsubsection{Using Textured Insoles of Different Heights}

This study explored the effect of using different height textured insoles on foot pressure in the patient. The results show that when a patient steps on an insole with a textured height, it will increase the pressure collected by each sensor due to the height of the particles. This is because although the subject has undergone some related operations to reduce edema, there is still some edema on the soles of the feet. Because of this, when we use a textured insole, it will increase the plantar pressure value due to the grain of the texture. The most obvious result of this is that when the particles increase from $1 \mathrm{~mm}$ to $3 \mathrm{~mm}$, or even to $6 \mathrm{~mm}$, the pressure value collected by each sensor increases significantly.

Therefore, when the patient uses the textured insole, the MP value (Figure 15) and PP value (Figure 16) of each contact point are also rising. However, it is interesting that as the values of MP and PP increase, the height of the textured insole does not increase in a proportional manner. In other words, when the insole height was increased from $3 \mathrm{~mm}$ to $6 \mathrm{~mm}$ texture, the increase in MP and PP did not double. This result may be because when the particle height increases, it more or less has a function similar to the arch of the foot. However, when the height reaches a certain level (for example, $3 \mathrm{~mm}$ ), increasing the height of the particles has a relatively limited effect on the angle of the arch of the foot. However, the exact reason for this discovery remains to be further systematically studied in the future. 

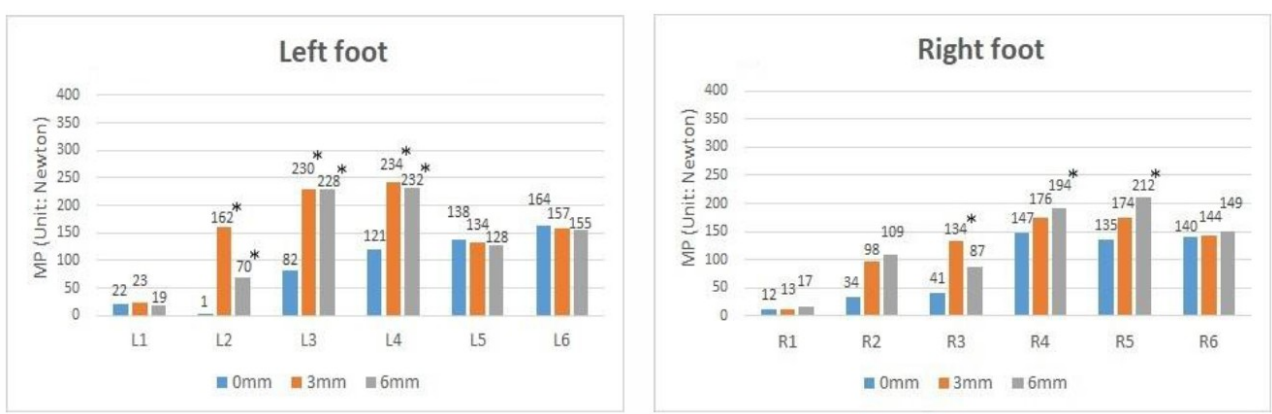

Figure 15. $\mathrm{MP}$ - effect of increasing texture height in the lymphedema patient. ${ }^{*} p$-value $<0.05$ indicates that a significant difference exists.
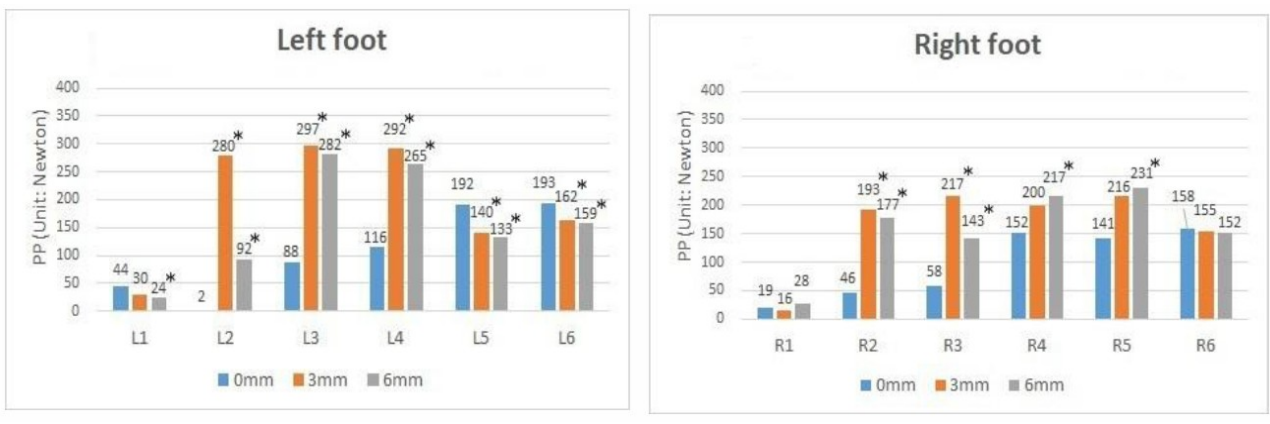

Figure 16. PP-effect of increasing texture height in the lymphedema patient. ${ }^{*} p$-value $<0.05$ indicates that a significant difference exists.

Another interesting finding is the effect of texture on the patient's maximum foot pressure from the perspective of PP value. From the perspective of the subject's left foot, it is obvious that when the texture height is increased, the pressure of his midfoot (L2, L3, L4) will increase. However, on the contrary, his forefoot (L1, L5, L6) and hind foot will decrease with the height of the texture. The result of the right foot is very similar to that of the left foot, but not so obvious. This is because the edema of the left foot is more serious than that of the right foot. Finally, from the STD point of view, when the height of the textured insole increases, the value of the patient's foot area (L2, L3, R2, R3) becomes very high (Figure 17). This result indicates that the use of textured insoles may cause considerable instability in midfoot pressure. From another perspective, the use of textured insoles may cause another injury to the midfoot.
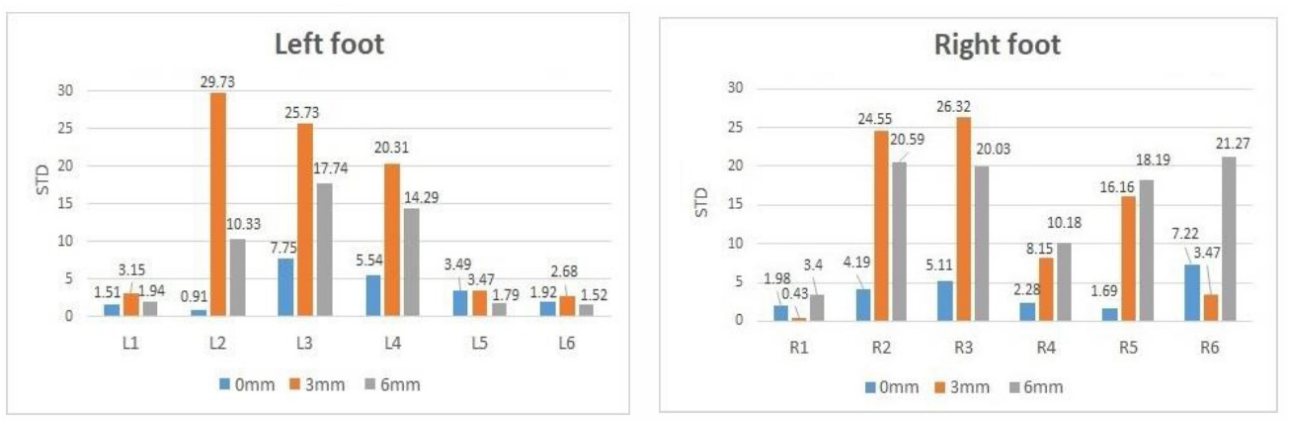

Figure 17. STD—effect of increasing texture height in the lymphedema patient.

\section{Discussion}

Whenever people stand, walk or perform sports, the human foot continues to withstand the reaction from the ground due to their bodyweight. Proper foot pressure distribution is critical to human health. When people have problems with their bodies or feet, this particular point concerns them greatly, and so this should not be neglected. 
Lymphedema is a type of chronic disease. The treatment of this disease is a long-term process. In this lengthy process, how to use non-invasive methods to provide patients with timely and appropriate plantar information is very important. On the recommendation of the doctor, there is an opportunity to invite a patient with lower extremity edema, overweight and plantar depression to participate in this study. During the study period of this article, this patient happened to need different lymphedema surgery. This provided the research team with the opportunity to use a set of self-made pressure sensor insoles to detect the patient's plantar pressure changes before and after the operation and use different insoles and arches to explore their impact on the patient.

At the appropriate time before and after the patient underwent the operation, the patient was invited to perform three simple daily activities: standing, walking and riding a bicycle. As far as the standing movement is concerned, this operation does significantly improve the plantar pressure at the corresponding points on the left and right feet of the patient with lymphedema. More importantly, it improves the patient's high plantar pressure in the midfoot and hindfoot area due to lower extremity edema. In terms of walking, it improves the patient's problems of too short stride and slow stride caused by lymphedema. From cycling activities, this study found that the operation can effectively improve the problem of high foot pressure in the foot block caused by cycling and the problem of high foot pressure in the flat thumb.

This study also used artificial foot arches to try to understand their effect on the patient's foot pressure. The results show that the artificial foot arch is effective in helping to alleviate the collapse problem, and can even provide moderate help to the patient. In addition, to our surprise, if a textured insole is used, not only will it not help improve the problem of arch collapse, but it may even increase the undesirable foot pressure due to the increased texture.

There are several inherent limitations in this study. First of all, the induction insole developed by this research institute is basically still a relatively simple device. In addition to its relatively limited functions, it may also produce some degree of error due to the interference of the surrounding environment. This is another limitation of the accuracy of the data collected in this research. In addition, due to the difference between the patient's own body and the average person to a certain extent, the collected information has a certain degree of uncertainty. Finally, due to the consideration of the patient's physical condition, the research team collects data without interfering with or hindering their physical condition, so the time and frequency of data collection are quite limited. However, it cannot be denied that these are only partial data, not a series of systematic data.

Finally, it is generally believed that lymphedema itself is a poorly understood disease. In the absence of complete data, the chance of a complete cure for patients is relatively small. In addition, the situation of each patient is different, and it is relatively difficult to analyze and research in a statistically comparative way. Under this limitation, the research results obtained from patients in this article can only be carried out in a case-by-case basis, and cannot be compared with other studies in relative terms.

\section{Conclusions}

The cost of the foot pressure-sensing system developed in this research team is about USD 200. There is a considerable gap between the price of commercial plantar pressure analysis systems currently on the market, which is between USD 10,000 and USD 20,000. The foot pressure-sensing system developed in this research is a self-test tool that most people can afford. In addition to cost considerations, it has great promotion value in clinical trials. There is no doubt that this foot pressure-sensing system can be applied to more patients with lower extremity edema, and even to diabetics and plantar flat patients. Another group of patients who may be suitable include those who need to put on cast shoes or foot supports due to foot surgery. In addition to increasing the applicable customer groups, a buzzer or sensor light can be added to the foot pressure-sensing system. When the system detects an abnormal phenomenon, the system can provide warning information 
to let the user know. Furthermore, the entire system can be connected to a personal mobile phone to send plantar information to the big data analysis system or provide relevant warning information at any time. When the entire system reaches a fairly mature stage, its application range will be wider.

Author Contributions: Conceptualization, J.-C.C., Y.-T.W. and Y.-S.L.; methodology, J.-C.C., Y.-T.W. and Y.-S.L.; software, J.-C.C. and Y.-T.W.; validation, J.-C.C., Y.-T.W. and Y.-S.L.; formal analysis, J.-C.C. and Y.-T.W.; investigation, J.-C.C. and Y.-T.W.; resources, J.-C.C. and Y.-T.W.; data curation, J.-C.C. and Y.-T.W.; writing-original draft preparation, J.-C.C. and Y.-T.W.; writing-review and editing, J.-C.C. and Y.-T.W.; visualization, J.-C.C. and Y.-T.W.; supervision, J.-C.C. and Y.-T.W.; project administration, J.-C.C.; funding acquisition, J.-C.C. and Y.-S.L. All authors have read and agreed to the published version of the manuscript.

Funding: This study was in part funded by the Taiwan Ministry of Science and Technology (Grant MOST 110-2221-E-224-041-MY3) and the National Taiwan University Hospital Yunlin Branch (Grant NTUHYL 107-C025).

Institutional Review Board Statement: The study was conducted according to the guidelines of the Declaration of Helsinki, and approved by the Ethics Committee of the National Taiwan University Hospital (No: 201805068 RINB, date: 18 June 2018).

Informed Consent Statement: Written informed consent has been obtained from the patient to publish this paper.

Data Availability Statement: Not applicable.

Acknowledgments: The authors would like to thank the approval of the Ethics Committee of National Taiwan University Hospital (No: 201805068 RINB, date: 18 June 2018).

Conflicts of Interest: The authors declare no conflict of interest.

\author{
Abbreviations \\ The following abbreviations are used in this manuscript: \\ AI arch index \\ MP mean pressure \\ PP peak pressure \\ STD standard deviation
}

\title{
References
}

1. Kaye, R.A.; Jahss, M.H. Tibialis posterior: A review of anatomy and biomechanics in relation to support of the medial longitudinal arch. Foot Ankle Int. 1991, 11, 244-247. [CrossRef]

2. Rome, K.; Mche, S.D. A study of the Properties of Materials Used in Podiatry. J. Am. Podiatr. Med Assoc. 1991, 81, 73-83. [CrossRef] [PubMed]

3. Cavanagh, P.R.; Bus, S.A. Off-loading the Diabetic Foot for Ulcer Prevention and Healing. J. Am. Podiatr. Med. Assoc. 2010, 100, 360-368. [CrossRef] [PubMed]

4. Macwilliams, B.A.; Cowley, M.; Nicholson, D.E. Foot kinematics and kinetics during adolescent gait. Gait Posture 2003, 17, 214-224. [CrossRef]

5. Keijsers, N.L.W.; Stolwijk, N.M.; Louwerens, J.W.K.; Duysens, J. Classification of forefoot pain based on plantar pressure measurements. Clin. Biomech. 2013, 28, 350-356. [CrossRef] [PubMed]

6. Orlin, M.N.; Mcpoil, T.G. Plantar pressure assessment. Phys. Ther. 2000, 80, 399-409. [CrossRef] [PubMed]

7. Kavros, S.J.; Straaten, M.G.V.; Wood, K.A.C.; Kaufman, K.R. Forefoot plantar pressure reduction of off-the-shelf rocker bottom provisional footwear. Clin. Biomech. 2011, 26, 778-782. [CrossRef] [PubMed]

8. Roy, K.J. Force, pressure and motion measurements in the foot: Current concepts. Clin. Podiatr. Med. Surg. 1988, 5, 491-508. [PubMed]

9. Birtane, M.; Tuna, H. The evaluation of plantar pressure distribution in obese and non-obese adults. Clin. Biomech. 2004, 19, 1055-1059. [CrossRef] [PubMed]

10. Sneyers, C.J.; Lysens, R.; Feys, H.; Andries, R. Influence of malaligment of foot on the plantar pressure pattern in running. Foot Ankle Int. 1995, 16, 624-632. [CrossRef] [PubMed]

11. Palya, Z.; Hampel, K.; Kiss, R.M. Lymphedema treatment's effect of gait parameters. Mater. Today Proc. 2018, 5, $26526-26531$. [CrossRef] 
12. Daniel, J.H.; Shuying, W.; Daniel, L.; Karen, V.; Chun, H.W.; Melissa, L.K.T. Comprehensive pressure profiling to develop next-generation compression treatment for lymphedema: Testing efficacy of high resolution sensors. Sens. Actuators A Phys. 2019, 289, 100-107.

13. Zuil-Escobar, J.C.; Martínez-Cepa, C.B.; Martín-Urrialde, J.A.; Gómez-Conesa, A. Reliability and Accuracy of Static Parameters Obtained from Ink and Pressure Platform Footprints. J. Manip. Physiol. Ther. 2016, 39, 510-517. [CrossRef] [PubMed]

14. Cavanagh, P.R.; Rodgers, M.M. The arch index: A useful measure from footprints. J. Biomech. 1987, 20, 547-551. [CrossRef]

15. Wang, Y.D.; Chen, J.C.; Lin, Y.S. Effects of Artificial Texture Insoles and Foot Arches on Improving Arch Collapse in Flat Feet. Sensors 2020, 20, 3667. [CrossRef] [PubMed] 